\title{
Perturbation Procedures in the Dynamic Analysis of a Toroidal Shell Segment Pressurized by a Step Load
}

\author{
Anthony Monday Ette, Joy Ulumma Chukwuchekwa*, Williams Ifeanyichukwu Osuji, \\ Atulegwu Chukwudi Osuji
}

Department of Mathematics, School of Physical Sciences, Federal University of Technology, Owerri, Imo State, Nigeria

Email address:

tonimonsette@yahoo.com (A. M. Ette), joyuchekwa@gmail.com (J. U. Chukwuchekwa), williams.osuji@futo.edu.ng (W. I. Osuji), aatac2000@gmail.com (A. C. Osuji)

${ }^{*}$ Corresponding author

\section{To cite this article:}

Anthony Monday Ette, Joy Ulumma Chukwuchekwa, Williams Ifeanyichukwu Osuji, Atulegwu Chukwudi Osuji. Perturbation Procedures in the Dynamic Analysis of a Toroidal Shell Segment Pressurized by a Step Load. American Journal of Mathematical and Computer Modelling. Vol. 5, No. 1, 2020, pp. 1-11. doi: 10.11648/j.ajmcm.20200501.11

Received: November 22, 2019; Accepted: December 13, 2019; Published: January 17, 2020

\begin{abstract}
This paper uses perturbation techniques in asymptotic procedures to determine the normal displacement, the associated Airy stress function and the dynamic buckling load of an imperfect, finite toroidal shell segment pressurized by a step load. The adoption of asymptotic and perturbation procedure is made possible by the presence of small non-dimensional parameter on which asymptotic expansions are made possible. It is assumed here that the imperfection can be regarded as the first term in the Fourier Sine series expansion. The buckling modes are also assumed to be strictly in the shape of the imperfection which is in turn given in the shape of the classical buckling mode. In the final analysis, a simple but implicit formula for determining the dynamic buckling load was obtained. The dynamic buckling load was related to the corresponding static buckling load and that relationship is independent of the imperfection parameter. It is observed, that this procedure, perhaps more than other ones, can be used to analyze relatively more complicated problems particularly where more demands and restrictions are placed on the imperfection parameter. The results are strictly and are valid as far as the imperfection parameter is relatively small compared to unity.
\end{abstract}

Keywords: Toroidal Shell, Airy Stress Function, Static and Dynamic Buckling, Asymptotic and Perturbation Technique

\section{Introduction}

An elastic toroidal shell segment is one of the most imperfection-sensitive structures in Structural Mechanics, yet, little (to our knowledge) seems to be known about its dynamic stability when subjected to time dependent loads. Unlike cylindrical shells which it shares some form of semblance of structural similarity, investigations probing into the dynamic behavior of toroidal shell segments appear to be rather scanty. In this investigation, our aim is to explore the deformation, in terms of the normal displacement and Airy stress function, of a finite imperfect toroidal shell segment pressurized by a step load. We shall also aim at deriving an implicit formula for evaluating the dynamic buckling load of the structure assuming that it is pressurized by a step load.

One of the earliest investigations on toroidal shell segments was done by Stein and McElman as in [1], while later, Hutchinson in [2] studied the initial post buckling behavior of toroidal shell segment. Some of the relatively recent studies on the structure include investigations by Oyesanya in [3, 4], who used perturbation techniques and asymptotics to study the static stability and imperfectionsensitivity of the structure when pressurized by a static load. Thus, except for a few of these investigations, there seems to be a dearth of strictly analytical studies on the dynamic behavior of the structure when loaded dynamically. However, over the years, there have been studies, in the dynamic realm, of similar structures including circular cylindrical and spherical shells as well as plates. Such investigations include those found in [5], that studied the dynamic buckling of externally pressurized imperfect cylindrical shells, as well as the work in [6], that studied nonlinear axisymmetric dynamic buckling of laminated angle-ply composite spherical caps. Other relevant studies include investigations in [7-11] among others. Mention must however be made of the investigation 
by Kriegesman as in [12], that investigated sample size dependent probabilistic design of axially compressed cylindrical shells, while in [13], Hu and Burgueño equally investigated elastic post buckling response of axially loaded cylindrical shells with seeded geometric imperfection design.

The aim of this work is to use regular perturbation technique and asymptotic expansion of the relevant variables to determine the normal displacement, the associated Airy stress function and the dynamic buckling load of an imperfect, finite toroidal shell segment pressurized by a step load. The work also intends to relate the dynamic buckling load to the corresponding static buckling load and that relationship is independent of the imperfection parameter.

\section{Formulation of Relevant Equations}

As in [3-4], the relevant Karman-Donnel equation of motion and compatibility equation regarding the normal displacement $\mathrm{W}(\mathrm{X}, \mathrm{Y}, \mathrm{T})$ and Airy stress function $\mathrm{F}(\mathrm{X}, \mathrm{Y}, \mathrm{T})$ of a finite imperfect toroidal shell segment of length $L$, pressurized by a step load $\mathrm{P}(\mathrm{T})$ are respectively given as

$$
\rho W_{, T T}+D \nabla^{4} W+\frac{1}{a} F_{, X X}+\frac{1}{b} F_{, Y Y}+P\left[\frac{1}{2}(W+\bar{W})_{, X X}+\left(1-\frac{1}{2} \frac{a}{b}\right)(W+\bar{W})_{, Y Y}\right]=S(W+\bar{W}, F)
$$

and

$$
\begin{gathered}
\frac{1}{E h} \nabla^{4} F-\frac{1}{a} W_{, X X}-\frac{1}{b} F_{, Y Y}=-\frac{1}{2} S(W+\bar{W}, W) \\
0<X<L, 0<Y<2 \pi \\
W=W_{, T}=0 \\
W=W_{, X X}=F=F_{, X X}=0 \text { at } X=0, L
\end{gathered}
$$

where, $\mathrm{E}$ is the Young's modulus and $\mathrm{X}$ and $\mathrm{Y}$ are the respective axial and circumferential coordinates, $\rho$ is the mass per unit area, $\mathrm{T}$ is the time variable, $\mathrm{a}$ and $\mathrm{b}$ are the inner and outer radii respectively, $\mathrm{h}$ is the thickness, $\mathrm{S}$ is a symmetric bilinear functional defined as

$$
S(P, Q)=P_{, X X} Q_{, Y Y}+P_{, Y Y} Q_{, Y Y}-2 P_{, X Y} Q_{, X Y}
$$

and $\nabla^{4}$ is the two-dimensional biharmonic operator defined as

$$
\nabla^{4} \equiv\left(\frac{\partial^{2}}{\partial X^{2}}+\frac{\partial^{2}}{\partial Y^{2}}\right)^{2}
$$

Here, $\bar{W}$ is the time-independent, stress-free and continuously differentiable imperfection function of $\mathrm{X}$ and $\mathrm{Y}$, while the bending stiffness $D$ is

$$
D=\frac{E h^{3}}{12\left(1-\vartheta^{2}\right)}
$$

where $\vartheta$ is Poisson's ratio.

\section{Nondimensionalization of the Governing Equations}

For convenience, we now introduce the following nondimensional quantities:

$$
\begin{gathered}
\bar{\nabla}^{4} w-K(\xi)\left(f_{, x x}+\xi r f_{, y y}\right)+\lambda\left[\frac{\alpha}{2}(w+\epsilon \bar{w})_{, x x}+\xi\left(1-\frac{\alpha}{2}\right)(w+\epsilon \bar{w})_{, y y}\right]=-K(\xi) H s(f, w+\epsilon \bar{w}) \\
\bar{\nabla}^{4} f-(1+\xi)^{2}\left(w_{, x x}+\xi r w_{, y y}\right)=-\frac{1}{2} H(1+\xi) s(w+\epsilon \bar{w}, w) \\
0<x<\pi, 0<y<2 \pi, t>0 \quad w=w_{, x x}=f=f_{, x x}=0 \text { at } x=0, \pi .
\end{gathered}
$$$$
w(x, y, 0)=w_{, t}(x, y, 0)=0
$$

$$
\begin{gathered}
x=\frac{\pi X}{L}, y=\frac{Y}{a}, \epsilon \bar{W}=\frac{\bar{W}}{h}, w=\frac{W}{h} \\
\lambda f(t)=\frac{L^{2} a P}{\pi^{2} D}, A=\frac{L^{2} \sqrt{12\left(1-\vartheta^{2}\right)}}{\pi^{2} a h}, H=\frac{h}{a} \\
\xi=\frac{L^{2}}{(\pi a)^{2}}, K(\xi)=-\left(\frac{A}{1+\xi}\right)^{2} \\
t=\frac{T \pi^{2}(D / \rho)^{1 / 2}}{L^{2}}
\end{gathered}
$$

Here, $\lambda$ is a nondimensional load amplitude whose value, $\lambda_{D}$ at dynamic buckling, we are to determine.

$P(t)$ is a step load such that

$$
P(t)=\left\{\begin{array}{l}
p, T>0 \\
0, T<0
\end{array}\right.
$$

while the imperfection amplitude $\epsilon$ is such that $0<\epsilon \ll 1$. We shall consider simply-supported boundary conditions as well as homogeneous initial conditions, and shall neglect boundary-layer effect by assuming that the pre-buckling deflection is constant so that we assume

$$
\begin{gathered}
F=-p a\left(X^{2}+\frac{1}{2} \alpha Y^{2}\right)+\frac{E h^{2} L^{2}}{\pi^{2} a(1+\xi)^{2}} f \\
W=\frac{p a^{2}(1-\alpha \nu)}{E h}+h w
\end{gathered}
$$

The first terms on the right hand sides of (13) and (14) are pre-buckling approximations while the parameter $\alpha$ takes the value $\alpha=1$ if pressure contributes to axial stress through end plates but $\alpha=0$ if pressure acts laterally.

On substituting all these quantities into (1) and (2) and simplifying, we get and 


$$
\bar{\nabla}^{4}=\left(\frac{\partial^{2}}{\partial x^{2}}+\xi \frac{\partial^{2}}{\partial y^{2}}\right)^{2}, s(p, q)=p_{, x x} q_{, y y}+p_{, y y} q_{, x x}-2 p_{, x y} q_{, x y}(20)
$$

A subscript following a comma indicates partial differentiation and $r=\frac{a}{b}$.

\section{Perturbation and Asymptotic Solution of the Problem}

We shall now let

$$
\hat{t}=\left(1+\mu \epsilon^{2}+\cdots\right) t
$$

$$
\therefore w_{, t}=\left(1+\mu \epsilon^{2}+\cdots\right) w_{, \hat{t}}, w_{, t t}=\left(1+\mu \epsilon^{2}+\cdots\right)^{2} w_{, \hat{t} \hat{t}}
$$

Now let

$$
\left(\begin{array}{l}
w \\
f
\end{array}\right)=\sum_{i=1}^{\infty}\left(\begin{array}{c}
w^{(i)} \\
f^{(i)}
\end{array}\right) \epsilon^{i}
$$

Substituting (23), (21) and (22) into (15) and (16) and equating the coefficients of powers of $\epsilon$, we get

$$
\begin{gathered}
O(\epsilon): w_{, t \hat{t}}^{(1)}+\bar{\nabla}^{4} w^{(1)}-K(\xi)\left(f_{, x x}^{(1)}+\xi r f_{, y y}^{(1)}\right)+\lambda\left[\frac{\alpha}{2}\left(w^{(1)}+\epsilon \bar{w}\right)_{, x x}+\xi\left(1-\frac{\alpha}{2}\right)\left(w^{(1)}+\epsilon \bar{w}\right)_{, y y}\right]=0 \\
O(\epsilon): \bar{\nabla}^{4} f^{(1)}-(1+\xi)^{2}\left(w_{, x x}^{(1)}+\xi r w_{, y y}^{(1)}\right)=0 \\
O\left(\epsilon^{2}\right): w_{, t \hat{t}}^{(2)}+\bar{\nabla}^{4} w^{(2)}-K(\xi)\left(f_{, x x}^{(2)}+\xi r f_{, y y}^{(2)}\right)+\lambda\left[\frac{\alpha}{2} w_{, x x}^{(2)}+\xi\left(1-\frac{\alpha}{2}\right) w_{, y y}^{(2)}\right] \\
=-K(\xi) H\left[s\left(f^{(1)}, w^{(1)}\right)+s\left(f^{(1)}, \bar{w}\right)\right] \\
O\left(\epsilon^{2}\right): \bar{\nabla}^{4} f^{(2)}-(1+\xi)^{2}\left(w_{, x x}^{(2)}+\xi r w_{, y y}^{(2)}\right)=-\frac{1}{2} H(1+\xi)\left[s\left(w^{(1)}, w^{(1)}\right)+s\left(w^{(1)}, \bar{w}\right)\right] \\
O\left(\epsilon^{3}\right): w_{, t \hat{t}}^{(2)}+\bar{\nabla}^{4} w^{(3)}-K(\xi)\left(f_{, x x}^{(3)}+\xi r f_{, y y}^{(3)}\right)+\lambda\left[\frac{\alpha}{2} w_{, x x}^{(3)}+\xi\left(1-\frac{\alpha}{2}\right) w_{, y y}^{(3)}\right] \\
=-K(\xi) H\left[s\left(f^{(1)}, w^{(2)}\right)+s\left(f^{(2)}, w^{(1)}\right)+s\left(f^{(2)}, \bar{w}\right)-2 \mu w_{, \hat{t} t}^{(1)}\right] \\
O\left(\epsilon^{3}\right): \bar{\nabla}^{4} f^{(3)}-(1+\xi)^{2}\left(w_{, x x}^{(3)}+\xi r w_{, y y}^{(3)}\right)=-\frac{1}{2} H(1+\xi)\left[s\left(w^{(1)}, w^{(2)}\right)+s\left(w^{(2)}, w^{(1)}\right)+s\left(w^{(2)}, \bar{w}\right)\right] \\
w^{(i)}=w_{, x x}^{(i)}=f^{(i)}=f_{, x x}^{(i)}=0, a t x=0, \pi \\
w(x, y, 0)=0, i=1,2,3, \ldots \\
w_{, t}^{(s)}(x, y, 0)=0, s=1,2 ; w_{, \hat{t}}^{(3)}(x, y, 0)+w_{, \hat{t}}^{(1)}(x, y, 0)=0
\end{gathered}
$$

etc.

The work in [5] had noted that any time-independent stress-free initial normal displacement $\bar{w}(x, y)$, satisfying reasonable smoothness conditions, can be expanded in a double Fourier series. Thus, if the edge effects are neglected and the origin of the circumferential coordinate is appropriately chosen, such a series is

$$
\bar{w}(x, y)=\bar{a} \sin x \sin n y+\sum_{\substack{m=1, k=0 \\(m, k) \neq(1, n)}}^{\infty}\left(\bar{a}_{m k} \sin k y+\bar{b}_{m k} \cos k y\right) \sin m x
$$

or

$$
\begin{gathered}
\bar{w}(x, y)=\sum_{m=1, k=0}^{\infty}\left(\bar{a}_{m k} \operatorname{sinky}+\bar{b}_{m k} \cos k y\right) \sin m x \\
\text { with } \\
\bar{b}_{1 n}=0
\end{gathered}
$$

However, for the purpose of the solution of the problem posed in this work, we shall assume

$$
\bar{w}(x, y)=\bar{a} \operatorname{sinm} x \sin n y
$$

where $\bar{a}$ is a scalar.

Throughout the analysis of this paper, we shall use the fact that, if 


$$
\begin{gathered}
w(x, y)=\sum_{p=1, q=0}^{\infty}\left(l_{1} \cos q y+l_{2} \sin q y\right) \sin p x \\
\text { then } \\
\bar{\nabla}^{4} w=\sum_{p=1, q=0}^{\infty}\left(p^{2}+\xi q^{2}\right)^{2}\left(l_{1} \cos q y+l_{2} \sin q y\right) \sin p x
\end{gathered}
$$

All through this analysis, any integration with respect to $\mathrm{x}$, will have 0 and $\pi$ as the lower and upper limits respectively while integration with respect to $y$ will have 0 and $2 \pi$ as the lower and upper limits respectively.

Solution of Equations of Order $\epsilon$

Generally, we shall let

$$
\left(\begin{array}{c}
w^{(i)} \\
f^{(i)}
\end{array}\right)=\sum_{p=1, q=0}^{\infty}\left(\begin{array}{c}
w_{1}^{(i)} \\
f_{1}^{(i)}
\end{array}\right) \sin p x \cos q y+\left(\begin{array}{c}
w_{2}^{(i)} \\
f_{2}^{(i)}
\end{array}\right) \operatorname{sinpx\operatorname {sin}qy}
$$

We now substitute (39) into (25), for $i=1$ and get, using (38)

$$
\begin{gathered}
\sum_{p=1, q=0}^{\infty}\left[\left\{\left(p^{2}+\xi q^{2}\right)^{2} f_{1}^{(1)}+(1+\xi)^{2}\left(q^{2} r \xi-p^{2}\right) w_{1}^{(1)}\right\} \sin p x \cos q y+\right. \\
\left.\left\{\left(p^{2}+\xi q^{2}\right)^{2} f_{2}^{(1)}+(1+\xi)^{2}\left(q^{2} r \xi-p^{2}\right) w_{2}^{(1)}\right\} \sin x x \sin q y\right]=0
\end{gathered}
$$

We now multiply (40) by sinm $x \cos n y$ and see that for $p=m, q=n$, we get

$$
f_{1}^{(1)}=\frac{-(1+\xi)^{2}\left(n^{2} r \xi-m^{2}\right) w_{1}^{(1)}}{\left(m^{2}+\xi r n^{2}\right)^{2}}
$$

Similarly, we next now multiply (39) by sinmxsinny and for $p=m, q=n$, integrate to get

$$
f_{2}^{(1)}=\frac{-(1+\xi)^{2}\left(n^{2} r \xi-m^{2}\right) w_{2}^{(1)}}{\left(m^{2}+\xi r n^{2}\right)^{2}}
$$

Next, we assume (39) in (24), multiply by sinmxsinny for $i=1$, and assume (36) and simplify to get (using (42))

$$
\begin{gathered}
w_{2, \hat{t} t}^{(1)}+\Psi^{2} w_{2}^{(1)}=\lambda \bar{a}\left\{\frac{\alpha m^{2}}{2}+n^{2} \xi\left(1-\frac{\alpha}{2}\right)\right\} \\
w_{2}^{(1)}(0)=w_{2, \hat{t}}^{(1)}(0)=0
\end{gathered}
$$

where,

$$
\Psi^{2}=\left[\left(m^{2}+\xi n^{2}\right)^{2}+\left\{\left(\frac{m A}{1+\xi}\right)^{2}+n^{2} r \xi\right\}(1+\xi)^{2}\left\{\frac{n^{2} r \xi-m^{2}}{\left(m^{2}+\xi n^{2}\right)^{2}}\right\}-\lambda\left\{\frac{\alpha m^{2}}{2}+\xi n^{2}\left(1-\frac{\alpha}{2}\right)\right\}\right]
$$

Here, we shall assume $\Psi^{2}>0, \forall m, n$. The solution of $(43-45)$ is

$$
\begin{aligned}
& w_{2}^{(1)}=B(1-\cos \Psi \hat{t}) \\
& B=\frac{\lambda \bar{a}\left(\frac{\alpha m^{2}}{2}+n^{2} \xi\left(1-\frac{\alpha}{2}\right)\right\}}{\Psi^{2}}
\end{aligned}
$$

We also multiply (39) by sinmxcosny for $i=1$, (assuming (36)), and simplify to get

$$
\begin{gathered}
w_{1, \hat{t} \hat{t}}^{(1)}+\Psi^{2} w_{1}^{(1)}=0 \\
w_{1}^{(1)}(0)=w_{1, \hat{t}}^{(1)}(0)=0
\end{gathered}
$$

The solution of (48) and (49) is

$$
w_{1}^{(1)} \equiv 0
$$

At this level of perturbation, we can write

$$
\left(\begin{array}{c}
w^{(1)} \\
f^{(1)}
\end{array}\right)=\left(\begin{array}{c}
1 \\
-\varphi_{0}
\end{array}\right) w_{2}^{(1)} \text { sinm } x \operatorname{sinn} y
$$

where, 


$$
\varphi_{0}=(1+\xi)^{2}\left\{\frac{n^{2} r \xi-m^{2}}{\left(m^{2}+n^{2} r \xi\right)^{2}}\right\}
$$

Solution of Equations of order $\epsilon^{2}$

On substituting for $w^{(1)}$ and $f^{(1)}$ from (51) and (52) in (26) and (27) and simplifying, we get

$$
\begin{aligned}
& w_{, \hat{t} \hat{t}}^{(2)}+\bar{\nabla}^{4} w^{(2)}-K(\xi)\left(f_{, x x}^{(2)}+\xi r f_{, y y}^{(2)}\right)+\lambda\left[\frac{\alpha m^{2}}{2} w_{, x x}^{(2)}+\xi\left(1-\frac{\alpha}{2}\right) w_{, y y}^{(2)}\right] \\
&=-K(\xi) H \varphi_{0}(m n)^{2}\left(w_{2}^{(1)^{2}}+\bar{a} w_{2}^{(1)}\right)(\cos 2 m x+\cos 2 n y) \\
& \bar{\nabla}^{4} f^{(2)}-(1+\xi)^{2}\left(w_{, x x}^{(2)}+\xi r w_{, y y}^{(2)}\right)=-\frac{1}{2} H(1+\xi)(m n)^{2}\left(w_{2}^{(1)^{2}}+\bar{a} w_{2}^{(1)}\right)(\cos 2 m x+\cos 2 n y)
\end{aligned}
$$

We now substitute (39) in (54), for $i=2$ and use (40) to get

$$
\begin{aligned}
\sum_{p=1, q=0}^{\infty}\left[\begin{array}{c}
\left\{\left(p^{2}+\xi q^{2}\right)^{2} f_{1}^{(2)}+(1+\xi)^{2}\left(q^{2} r \xi-p^{2}\right) w_{1}^{(2)}\right\} \sin p x \cos q y \\
+\left\{\left(p^{2}+\xi q^{2}\right)^{2} f_{2}^{(2)}+(1+\xi)^{2}\left(q^{2} r \xi-p^{2}\right) w_{2}^{(2)}\right\} \sin p x \sin q y
\end{array}\right] \\
=-\frac{1}{2} H(1+\xi)(m n)^{2}\left(w_{2}^{(1)^{2}}+\bar{a} w_{2}^{(1)}\right)(\cos 2 m x+\cos 2 n y)
\end{aligned}
$$

Multiplying (55) by $\operatorname{sinm} x \cos 2 n y$ and for $p=m$, (m odd), $q=2 n$, we get

$$
f_{1}^{(2)}=\frac{\frac{-2 H(1+\xi) m n^{2}}{\pi}\left(w_{2}^{(1)^{2}}+\bar{a} w_{2}^{(1)}\right)-(1+\xi)^{2}\left(4 n^{2} r \xi-m^{2}\right) w_{1}^{(2)}}{\left(m^{2}+4 n^{2} \xi\right)^{2}}
$$

We can further write

$$
\begin{aligned}
& f_{1}^{(2)}=-\varphi_{11}\left(w_{2}^{(1)^{2}}+\bar{a} w_{2}^{(1)}\right)-\varphi_{12} w_{1}^{(2)} \\
& \varphi_{11}=\frac{\frac{2 H(1+\xi) m n^{2}}{\pi}}{\left(m^{2}+4 n^{2} \xi\right)^{2}}, \varphi_{12}=\frac{(1+\xi)^{2}\left(4 n^{2} r \xi-m^{2}\right)}{\left(m^{2}+4 n^{2} \xi\right)^{2}}
\end{aligned}
$$

Similarly, multiplying (56) by $\sin m x \sin 2 n y$ and for $p=m$, (m odd), $q=2 n$, we get

$$
f_{2}^{(2)}=\frac{-(1+\xi)^{2}\left(4 n^{2} r \xi-m^{2}\right) w_{2}^{(2)}}{\left(m^{2}+4 n^{2} r \xi\right)^{2}}
$$

Next, we substitute in (53) and thereafter, multiply through by $\operatorname{sinm} x \cos 2 n y$, and for $p=m$, (m odd), $q=2 n$, simplify to get (using (56))

$$
\begin{gathered}
w_{1, \hat{t} \hat{t}}^{(2)}+\Omega^{2} w_{1}^{(2)}=-\left(\varphi_{2}+\varphi_{3}\right)\left(w_{2}^{(1)^{2}}+\bar{a} w_{2}^{(1)}\right) \\
w_{1}^{(2)}(0)=w_{1, \hat{t}}^{(2)}(0)=0
\end{gathered}
$$

where,

$$
\begin{gathered}
\Omega^{2}=\left[\left(m^{2}+4 \xi n^{2}\right)^{2}+\left\{\left(\frac{m A}{1+\xi}\right)^{2}+4 n^{2} \xi\right\}(1+\xi)^{2}\left\{\frac{4 n^{2} r \xi-m^{2}}{\left(m^{2}+4 n^{2} \xi\right)^{2}}\right\}-\lambda\left\{\frac{\alpha m^{2}}{2}+4 n^{2} \xi\left(1-\frac{\alpha}{2}\right)\right\}\right]>0, \forall m, n \\
\varphi_{2}=\left\{\left(\frac{m A}{1+\xi}\right)^{2}+4 n^{2} \xi\right\}\left\{\frac{2 H(1+\xi) m n^{2}}{\left(m+4 n^{2} \xi\right) \pi}\right\} \\
\varphi_{3}=\frac{4 \varphi_{0} H m n^{2}}{\pi}\left(\frac{A}{1+\xi}\right)^{2}
\end{gathered}
$$

In the same fashion, we next multiply (53) through by sinm $x \sin 2 n y$ and simplify to get

$$
\begin{gathered}
w_{2, \hat{t} \hat{t}}^{(2)}+\Omega^{2} w_{2}^{(2)}=0 \\
w_{2}^{(2)}(0)=w_{2, \hat{t}}^{(2)}(0)=0
\end{gathered}
$$


Using (46), it is seen that (58) simplifies to

$$
w_{1, \hat{t} \hat{t}}^{(2)}+\Omega^{2} w_{1}^{(2)}=-\varphi_{4}\left[\varphi_{5}-\varphi_{6} \cos \Psi \hat{t}+\varphi_{7} \cos 2 \Psi \hat{t}\right]
$$

where,

$$
\begin{gathered}
\varphi_{4}=\varphi_{2}+\varphi_{3}, \varphi_{5}=\left(\frac{3}{2} B^{2}+\bar{a} B\right) \\
\varphi_{6}=\left(2 B^{2}+\bar{a} B\right), \varphi_{7}=\frac{B^{2}}{2}
\end{gathered}
$$

Solving (67 - 69) yields

$$
w_{1}^{(2)}=\beta_{1} \cos \Omega \hat{t}-\varphi_{4}\left[\frac{\varphi_{5}}{\Omega^{2}}-\frac{\varphi_{6} \cos \psi \hat{t}}{\Omega^{2}-\Psi^{2}}+\frac{\varphi_{7} \cos 2 \Psi \hat{t}}{\Omega^{2}-4 \Psi^{2}}\right]
$$

where,

$$
\begin{gathered}
\beta_{1}=\varphi_{4}\left[\frac{\varphi_{5}}{\Omega^{2}}-\frac{\varphi_{6}}{\Omega^{2}-\Psi^{2}}+\frac{\varphi_{7}}{\Omega^{2}-4 \Psi^{2}}\right] \\
\beta_{1}=B^{2} \varphi_{4}\left[\frac{3}{2 \Omega^{2}}-\frac{2}{\Omega^{2}-\Psi^{2}}+\frac{1}{2\left(\Omega^{2}-4 \Psi^{2}\right)}\right]+O(B)
\end{gathered}
$$

Similarly, the solution of (65) and (66) is

$$
w_{2}^{(2)} \equiv 0
$$

It follows at this order of perturbation, that

$$
\left(\begin{array}{c}
w^{(2)} \\
f^{(2)}
\end{array}\right)=\left(\begin{array}{c}
w_{1}^{(2)} \\
f_{1}^{(2)}
\end{array}\right) \sin m x \cos 2 n y
$$

Solutions of Equations of Order $\epsilon^{3}$

We now substitute in (28) and (29) to get

$$
\begin{gathered}
w_{, \hat{t} \hat{t}}^{(3)}+\bar{\nabla}^{4} w^{(3)}-K(\xi)\left(f_{, x x}^{(2)}+\xi r f_{, y y}^{(2)}\right)+\lambda\left[\frac{\alpha m^{2}}{2} w_{, x x}^{(3)}+\xi\left(1-\frac{\alpha}{2}\right) w_{, y y}^{(3)}\right] \\
=-(m n)^{2} K(\xi) H[9 \sin 3 n y-\sin n y-\cos 2 m x \sin 3 n y+9 \cos 2 m x \sin n y]\left(2 w_{2}^{(1)} w_{1}^{(2)}+\bar{a} w_{2}^{(2)}\right)-2 \mu_{2} w_{2, \hat{t} \hat{t}}^{(1)} \sin m x \operatorname{sinn} y \\
\bar{\nabla}^{4} f^{(3)}-(1+\xi)^{2}\left(w_{, x x}^{(3)}+\xi r w_{, y y}^{(3)}\right)=-\frac{1}{8}(m n)^{2} H(1+\xi)[9 \sin 3 n y-\sin n y-\cos 2 m x \sin 3 n y+9 \cos 2 m x \operatorname{sinn} y]\left(2 w_{2}^{(1)} w_{1}^{(2)}+\bar{a} w_{2}^{(2)}\right)
\end{gathered}
$$

Now, let

$$
\left(\begin{array}{c}
w^{(3)} \\
f^{(3)}
\end{array}\right)=\sum_{p=1, q=0}^{\infty}\left(\begin{array}{c}
w_{1}^{(3)} \\
f_{1}^{(3)}
\end{array}\right) \sin p x \cos q y+\left(\begin{array}{c}
w_{2}^{(3)} \\
f_{2}^{(3)}
\end{array}\right) \sin p x \sin q y
$$

Substituting (76) in (75), gives

$$
\begin{gathered}
\sum_{p=1, q=0}^{\infty}\left[\begin{array}{c}
\left\{\left(p^{2}+\xi q^{2}\right)^{2} f_{1}^{(3)}+(1+\xi)^{2}\left(q^{2} r \xi-p^{2}\right) w_{1}^{(3)}\right\} \operatorname{sinpxcos} q y \\
+\left\{\left(p^{2}+\xi q^{2}\right)^{2} f_{2}^{(3)}+(1+\xi)^{2}\left(q^{2} r \xi-p^{2}\right) w_{2}^{(3)}\right\} \sin p x \sin q y
\end{array}\right] \\
=-\frac{1}{8}(m n)^{2} H(1+\xi)[9 \sin 3 n y-\sin n y-\cos 2 m x \sin 3 n y+9 \cos 2 m x \sin n y]\left(2 w_{2}^{(1)} w_{1}^{(2)}+\bar{a} w_{2}^{(2)}\right)
\end{gathered}
$$

Next, we multiply (77) by sinmxsinny and for $p=m$ (odd), $q=n$, get

$$
f_{2(n)}^{(3)}=\frac{H(1+\xi) m n^{2}\left(2 w_{2}^{(1)} w_{1}^{(2)}+\bar{a} w_{1}^{(2)}\right)}{2 \pi\left(m^{2}+n^{2} \xi\right)^{2}}-\frac{(1+\xi)^{2}\left(n^{2} r \xi-m^{2}\right) w_{2(n)}^{(3)}}{\left(m^{2}+n^{2} \xi\right)^{2}}
$$

In the same way, multiplying (77) by $\sin m x \sin 3 n y$, we get (after simplification) 


$$
f_{2(3 n)}^{(3)}=-\left[\frac{9 m n^{2} H(1+\xi)\left(2 w_{2}^{(1)} w_{1}^{(2)}+\bar{a} w_{1}^{(2)}\right)}{2 \pi\left(m^{2}+9 n^{2} \xi\right)^{2}}+\frac{(1+\xi)^{2}\left(9 n^{2} r \xi-m^{2}\right) w_{2(3 n)}^{(3)}}{\left(m^{2}+9 n^{2} \xi\right)^{2}}\right]
$$

Let

$$
\begin{gathered}
\varphi_{8}=\frac{H(1+\xi) m n^{2}}{2 \pi\left(m^{2}+n^{2} \xi\right)^{2}} \\
\varphi_{9}=\frac{9 H(1+\xi) m n^{2}}{2 \pi\left(m^{2}+9 n^{2} \xi\right)^{2}}, \varphi_{10}=\frac{(1+\xi)^{2}\left(9 n^{2} r \xi-m^{2}\right)}{\left(m^{2}+9 n^{2} \xi\right)^{2}} \\
\therefore f_{2(n)}^{(3)}=\varphi_{8}\left(2 w_{2}^{(1)} w_{1}^{(2)}+\bar{a} w_{1}^{(2)}\right)-\varphi_{0} w_{2(n)}^{(3)}
\end{gathered}
$$

and

$$
f_{2(3 n)}^{(3)}=-\left[\varphi_{9}\left(2 w_{2}^{(1)} w_{1}^{(2)}+\bar{a} w_{1}^{(2)}\right)+\varphi_{10} w_{2(3 n)}^{(3)}\right]
$$

We next substitute for terms in (74), multiply through by sinmxsinny and for $p=m, q=n$, get

$$
w_{2(n), \hat{t} \hat{t}}^{(3)}+\Psi^{2} w_{2(n)}^{(3)}=\frac{-H K m n^{2}}{\pi}\left(f_{2}^{(1)} w_{1}^{(2)}+f_{1}^{(2)} w_{2}^{(1)}+f_{1}^{(2)} \bar{a}\right)+\frac{H(1+\xi) m n^{2}}{2 \pi\left(m^{2}+n^{2} \xi\right)^{2}}\left\{\left(\frac{m A}{1+\xi}\right)^{2}+n^{2} r \xi\right\}\left(2 w_{2}^{(1)} w_{1}^{(2)}+\bar{a} w_{1}^{(2)}\right)-2 \mu w_{2, \bar{t} \bar{t}}^{(1)}
$$

By means of simplification, it is to be noted that the first bracket on the right hand side of (84) can be simplified to read

$$
\frac{-H K m n^{2}}{\pi}\left(f_{2}^{(1)} w_{1}^{(2)}+f_{1}^{(2)} w_{2}^{(1)}+f_{1}^{(2)} \bar{a}\right)=-\varphi_{13}\left[\left(\varphi_{12}+\varphi_{0}\right) w_{1}^{(2)} w_{2}^{(1)}+\varphi_{11} w_{2}^{(1)}{ }^{3}+2 \bar{a} \varphi_{11} w_{2}^{(1)^{2}}+\bar{a}^{2} \varphi_{11} w_{2}^{(1)}+\bar{a} \varphi_{12} w_{1}^{(2)}\right]
$$

where,

$$
\begin{gathered}
\varphi_{13}=\frac{H K m n^{2}}{\pi}\left(\frac{A}{1+\xi}\right)^{2} \\
\text { Let } \\
\varphi_{14}=\frac{H(1+\xi) m n^{2}}{2 \pi\left(m^{2}+n^{2} \xi\right)^{2}}\left\{\left(\frac{m A}{1+\xi}\right)^{2}+n^{2} r \xi\right\}
\end{gathered}
$$

In other word, we can recast (84) as

$$
\begin{gathered}
w_{2(n), \hat{t} \hat{t}}^{(3)}+\Psi^{2} w_{2(n)}^{(3)}=-\varphi_{13}\left[\left(\varphi_{12}+\varphi_{0}\right) w_{1}^{(2)} w_{2}^{(1)}+\varphi_{11} w_{2}^{(1)^{3}}+2 \bar{a} \varphi_{11} w_{2}^{(1)^{2}}+\bar{a}^{2} \varphi_{0} w_{2}^{(1)}+\bar{a} \varphi_{12} w_{1}^{(2)}\right] \\
+\varphi_{14}\left(2 w_{1}^{(2)} w_{2}^{(1)}+\bar{a} w_{1}^{(2)}\right)-2 \mu w_{2, \bar{t} \hat{t}}^{(1)} \\
w_{2(n)}^{(3)}(0)=0, w_{2(n), \hat{t}}^{(3)}(0)+w_{2(n), \hat{t}}^{(1)}(0)=0
\end{gathered}
$$

We can further simplify $(88)$ to read

$$
\begin{gathered}
w_{2(n), \hat{t} \hat{t}}^{(3)}+\Psi^{2} w_{2(n)}^{(3)}=\left\{2 \varphi_{14}-\varphi_{13}\left(\varphi_{12}+\varphi_{0}\right)\right\} w_{1}^{(2)} w_{2}^{(1)}+\bar{a} w_{1}^{(2)}\left\{\varphi_{14}-\varphi_{13} \varphi_{12}\right\}- \\
\varphi_{13}\left\{\varphi_{11} w_{2}^{(1)^{3}}+2 \bar{a} \varphi_{11} w_{2}^{(1)^{2}}+\bar{a}^{2} \varphi_{0} w_{2}^{(1)}\right\}-2 \mu w_{2, \hat{t} \hat{t}}^{(1)}
\end{gathered}
$$

Certain terms appearing in (90) can be simplified as follows:

$$
\begin{gathered}
w_{1}^{(2)} w_{2}^{(1)}=B^{3} \varphi_{4}\left[\begin{array}{c}
\left(\frac{3}{2 \Omega^{2}}-\frac{1}{\Omega^{2}-\Psi^{2}}\right)+\left\{\frac{2}{\Omega^{2}-\Psi^{2}}+\frac{3}{2 \Omega^{2}}+\frac{1}{4\left(\Omega^{2}-4 \Psi^{2}\right)}\right\} \cos \Psi \hat{t}- \\
\left\{\frac{1}{2\left(\Omega^{2}-4 \Psi^{2}\right)}+\frac{1}{\Omega^{2}-\Psi^{2}}\right\} \cos 2 \Psi \hat{t}+\left(\frac{1}{4\left(\Omega^{2}-4 \Psi^{2}\right)}\right) \cos 3 \Psi \hat{t}
\end{array}\right] \\
+B^{3} \varphi_{16}\left\{\cos \Omega \hat{t}-\frac{1}{2}(\cos (\Omega+\Psi) \hat{t}+\cos (\Omega-\Psi) \hat{t})\right\}+O\left(B^{2}\right) \\
w_{2}^{(1)^{3}}=B^{3}\left[\frac{5}{2}-\frac{15}{4} \cos \Psi \hat{t}+\frac{3}{2} \cos 2 \Psi \hat{t}-\frac{\cos 3 \Psi \hat{t}}{4}\right] \\
w_{2}^{(1)^{2}}=B^{2}\left[\frac{3}{2}-2 \cos \Psi \hat{t}+\frac{\cos 2 \Psi \hat{t}}{2}\right]
\end{gathered}
$$


where,

$$
\varphi_{16}=\left[\frac{3}{2 \Omega^{2}}-\frac{2}{\Omega^{2}-\Psi^{2}}+\frac{1}{2\left(\Omega^{2}-4 \Psi^{2}\right)}\right]
$$

We now substitute all the simplified terms into (90) and to ensure a uniformly valid solution in $\hat{t}$, equate to zero the coefficient of $\cos \Psi \hat{t}$ and get

$$
\mu=\frac{B^{2}}{2 \Psi^{2}}\left[\frac{15 \varphi_{11} \varphi_{13}}{4}-\varphi_{4}\left\{2 \varphi_{14}-\varphi_{13}\left(\varphi_{12}+\varphi_{0}\right)\right\}\left\{\frac{2}{\Omega^{2}-\Psi^{2}}+\frac{3}{2 \Omega^{2}}+\frac{1}{4\left(\Omega^{2}-4 \psi^{2}\right)}\right\}\right]+O(B)
$$

The remaining equation in (90) is re-arranged to give

$$
\begin{gathered}
w_{2(n), \hat{t} \hat{t}}^{(3)}+\Psi^{2} w_{2(n)}^{(3)}=B^{3}\left[\varphi_{17}+\varphi_{18} \cos 2 \Psi \hat{t}+\varphi_{19} \cos 3 \Psi \hat{t}+\varphi_{20} \cos \Omega \hat{t}+\varphi_{21} \cos (\Psi+\Omega) \hat{t}+\varphi_{22} \cos (\Psi-\Omega) \hat{t}\right] \\
w_{2(n)}^{(3)}(0)=0, w_{2(n), \hat{t}}^{(3)}(0)+w_{2(n), \hat{t}}^{(1)}(0)=0
\end{gathered}
$$

where,

$$
\begin{gathered}
\varphi_{17}=\varphi_{15}\left(\frac{3}{2 \Omega^{2}}-\frac{1}{\Omega^{2}-\Psi^{2}}\right)-\frac{5 \varphi_{11} \varphi_{13}}{2} \\
\varphi_{18}=-\varphi_{15}\left(\frac{1}{2\left(\Omega^{2}-4 \psi^{2}\right)}+\frac{1}{\Omega^{2}-\Psi^{2}}\right)-\frac{3 \varphi_{11} \varphi_{13}}{2} \\
\varphi_{19}=\frac{\varphi_{15}}{4}\left(\frac{1}{\Omega^{2}-4 \Psi^{2}}\right)+\frac{\varphi_{11} \varphi_{13}}{4} \\
\varphi_{20}=\varphi_{15} \varphi_{16}, \varphi_{21}=\frac{-\varphi_{15} \varphi_{16}}{2}=\varphi_{22}
\end{gathered}
$$

The solution of (96) and (97) is

$$
\begin{gathered}
w_{2(n)}^{(3)}(\hat{t})=\beta_{2} \cos \Psi \hat{t}+B^{3}\left[\frac{\varphi_{17}}{\psi^{2}}-\frac{\varphi_{18} \cos 2 \Psi \hat{t}}{3 \Psi^{2}}-\frac{\varphi_{19} \cos 3 \Psi \hat{t}}{8 \Psi^{2}}+\frac{\varphi_{20} \cos \Omega \hat{t}}{\Psi^{2}-\Omega^{2}}-\frac{\varphi_{21} \cos (\Psi+\Omega) \hat{t}}{\Omega(2 \Psi+\Omega)}+\frac{\varphi_{22} \cos (\Psi-\Omega) \hat{t}}{\Omega(2 \Psi-\Omega)}\right] \\
\beta_{2}=-B^{3}\left[\frac{\varphi_{17}}{\Psi^{2}}-\frac{\varphi_{18}}{3 \Psi^{2}}-\frac{\varphi_{19}}{8 \Psi^{2}}+\frac{\varphi_{20}}{\Psi^{2}-\Omega^{2}}-\frac{\varphi_{21}}{\Omega(2 \Psi+\Omega)}+\frac{\varphi_{22}}{\Omega(2 \Psi-\Omega)}\right]
\end{gathered}
$$

We now substitute into (77), multiply by $\sin m x \sin 3 n y$, and for $p=m($ odd $), q=3 n$, arrange to get

$$
\begin{gathered}
w_{2(3 n), \hat{t} \hat{t}}^{(3)}+\omega^{2} w_{2(3 n)}^{(3)}=-\varphi_{23}\left(f_{2}^{(1)} w_{1}^{(2)}+f_{1}^{(2)}\left(w_{2}^{(1)}+\bar{a}\right)\right)+\varphi_{24}\left(2 w_{2}^{(1)} w_{1}^{(2)}+\bar{a} w_{1}^{(2)}\right) \\
w_{2(3 n)}^{(3)}(0)=0, w_{2(3 n), \hat{t}}^{(3)}(0)=0
\end{gathered}
$$

where,

$$
\omega^{2}=\left[\left(m^{2}+9 n^{2} \xi\right)^{2}+\left\{\left(\frac{m A}{1+\xi}\right)^{2}+9 n^{2} \xi r\right\}(1+\xi)^{2}\left(9 n^{2} \xi r-m^{2}\right)-\lambda\left\{\frac{\alpha m^{2}}{2}+9 n^{2} \xi\left(1-\frac{\alpha}{2}\right)\right\}\right]>0, \forall m, n(10
$$

where,

$$
\varphi_{23}=\frac{H\left(\frac{A}{1+\xi}\right)^{2} m n^{2}}{\pi}, \varphi_{24}=\frac{9 H m n^{2}(1+\xi)^{2}}{2 \pi}\left\{\left(\frac{m A}{1+\xi}\right)^{2}+9 n^{2} \xi r\right\}
$$

The first term on the right hand side of (104) can be simplified as

$$
-\varphi_{23}\left(f_{2}^{(1)} w_{1}^{(2)}+f_{1}^{(2)}\left(w_{2}^{(1)}+\bar{a}\right)\right)=-\varphi_{23}\left[\left(\varphi_{12}+\varphi_{0}\right) w_{1}^{(2)} w_{2}^{(1)}+\varphi_{11} w_{2}^{(1)^{3}}+2 \bar{a} w_{2}^{(1)^{2}}+\bar{a}^{2} \varphi_{11} w_{2}^{(1)}+\bar{a} \varphi_{12} w_{1}^{(2)}\right]
$$

Thus, equation (101) takes the form

$$
w_{2(3 n), \hat{t} \hat{t}}^{(3)}+\omega^{2} w_{2(3 n)}^{(3)}=-\varphi_{23}\left[\left(\varphi_{12}+\varphi_{0}\right) w_{1}^{(2)} w_{2}^{(1)}+\varphi_{11} w_{2}^{(1)^{3}}+2 \bar{a} w_{2}^{(1)}{ }^{2}+\bar{a}^{2} \varphi_{11} w_{2}^{(1)}+\bar{a} \varphi_{12} w_{1}^{(2)}\right]+\varphi_{24}\left(2 w_{2}^{(1)} w_{1}^{(2)}+\bar{a} w_{1}^{(2)}\right)
$$

In passing, we note that the term $w_{1}^{(2)} w_{2}^{(1)}$ appearing in (108) is as already simplified in (91). We further simplify (108) to read 


$$
w_{2(3 n), \hat{t} \hat{t}}^{(3)}+\omega^{2} w_{2(3 n)}^{(3)}=\left[\left\{2 \varphi_{24}-\varphi_{23}\left(\varphi_{12}+\varphi_{0}\right)\right\} w_{1}^{(2)} w_{2}^{(1)}+\bar{a} w_{1}^{(2)}\left\{\varphi_{24}-\varphi_{23} \varphi_{12}\right\}-\varphi_{23}\left\{\varphi_{11} w_{2}^{(1)^{3}}+2 \bar{a} w_{2}^{(1)^{2}}+\bar{a}^{2} \varphi_{0} w_{2}^{(1)}\right\}\right](10
$$

Yet, a final simplification of (110) is

$$
\begin{gathered}
w_{2(3 n), \hat{t} \hat{t}}^{(3)}+\omega^{2} w_{2(3 n)}^{(3)}=B^{3}\left[\varphi_{25}+\varphi_{26} \cos \Psi \hat{t}+\varphi_{27} \cos 2 \Psi \hat{t}+\varphi_{28} \cos 3 \Psi \hat{t}+\varphi_{29} \cos \Omega \hat{t}+\varphi_{30} \cos (\Psi+\Omega) \hat{t}+\varphi_{31} \cos (\Psi-\Omega) \hat{t}\right] \\
w_{2(3 n)}^{(3)}(0)=0, w_{2(3 n), \hat{t}}^{(3)}(0)=0
\end{gathered}
$$

where,

$$
\begin{gathered}
\varphi_{25}=\varphi_{15}\left(\frac{3}{2 \Omega^{2}}-\frac{1}{\Omega^{2}-\Psi^{2}}\right)-\frac{5 \varphi_{11} \varphi_{23}}{2} \\
\varphi_{26}=\left[\frac{2}{\Omega^{2}-\Psi^{2}}+\frac{3}{2 \Omega^{2}}+\frac{1}{4\left(\Omega^{2}-4 \Psi^{2}\right)}-\frac{15}{4}\right]+O\left(B^{2}\right) \\
\varphi_{27}=-\varphi_{15}\left(\frac{1}{2\left(\Omega^{2}-4 \Psi^{2}\right)}+\frac{1}{\Omega^{2}-\Psi^{2}}\right)-\frac{3 \varphi_{11} \varphi_{23}}{2} \\
\varphi_{28}=\frac{\varphi_{15}}{4}\left(\frac{1}{\Omega^{2}-4 \Psi^{2}}\right)+\frac{\varphi_{11} \varphi_{23}}{4}, \varphi_{29}=\varphi_{15} \varphi_{16} \\
\varphi_{30}=\varphi_{31}=\frac{-\varphi_{15} \varphi_{16}}{2}
\end{gathered}
$$

Solving (111) and (112), gives

$$
\begin{gathered}
w_{2(3 n)}^{(3)}(\hat{t})=\beta_{3} \cos \omega \hat{t}+B^{3}\left[\frac{\varphi_{25}}{\omega^{2}}+\frac{\varphi_{26} \cos \Psi \hat{t}}{\omega^{2}-\Psi^{2}}+\frac{\varphi_{27} \cos 2 \Psi \hat{t}}{\omega^{2}-4 \psi^{2}}+\frac{\varphi_{28} \cos 3 \Psi \hat{t}}{\omega^{2}-9 \Psi^{2}}+\frac{\varphi_{29} \cos \Omega \hat{t}}{\omega^{2}-\Omega^{2}}+\frac{\varphi_{30} \cos (\Psi+\Omega) \hat{t}}{\omega^{2}-(\Psi+\Omega)^{2}}+\frac{\varphi_{31} \cos (\Psi-\Omega) \hat{t}}{\omega^{2}-(\Psi-\Omega)^{2}}\right] \\
\beta_{3}=-B^{3}\left[\frac{\varphi_{25}}{\omega^{2}}+\frac{\varphi_{26}}{\omega^{2}-\Psi^{2}}+\frac{\varphi_{27}}{\omega^{2}-4 \Psi^{2}}+\frac{\varphi_{28}}{\omega^{2}-9 \Psi^{2}}+\frac{\varphi_{29}}{\omega^{2}-\Omega^{2}}+\frac{\varphi_{30}}{\omega^{2}-(\Psi+\Omega)^{2}}+\frac{\varphi_{31}}{\omega^{2}-(\Psi-\Omega)^{2}}\right]
\end{gathered}
$$

At this level of perturbation, we write

$$
\left(\begin{array}{c}
w^{(3)} \\
f^{(3)}
\end{array}\right)=\left(\begin{array}{c}
w_{2(n)}^{(3)} \\
f_{2(n)}^{(3)}
\end{array}\right) \sin m x \operatorname{sinn} y+\left(\begin{array}{c}
w_{2(3 n)}^{(3)} \\
f_{2(3 n)}^{(3)}
\end{array}\right) \sin m x \sin 3 y
$$

Thus, generally, the entire deformation, in terms of displacement and the associated Airy stress function, is

$$
\left(\begin{array}{l}
w \\
f
\end{array}\right)=\epsilon\left(\begin{array}{c}
w_{2}^{(1)} \\
f_{2}^{(1)}
\end{array}\right) \operatorname{sinm} x \operatorname{sinn} y+\epsilon^{2}\left(\begin{array}{c}
w_{1}^{(2)} \\
f_{1}^{(2)}
\end{array}\right) \operatorname{sinm} x \cos 2 y+\epsilon^{3}\left[\left(\begin{array}{c}
w_{2(n)}^{(3)} \\
f_{2(n)}^{(3)}
\end{array}\right) \sin m x \operatorname{sinn} y+\left(\begin{array}{c}
w_{2(3 n)}^{(3)} \\
f_{2(3 n)}^{(3)}
\end{array}\right) \sin m x \sin 3 n y\right]+\cdots(11
$$

\section{Dynamic Buckling Load}

To determine the dynamic buckling load $\lambda_{D}$, we shall use the displacement components that are strictly in the shape of imperfection. Thus, from (119), we have

$$
w=\epsilon w_{2}^{(1)} \operatorname{sinm} x \sin n y+\epsilon^{3} w_{2(n)}^{(3)} \operatorname{sinm} x \sin n y+\cdots(
$$

As observed by $[14,15]$, the condition for dynamic buckling load is

$$
\frac{d \lambda}{d w_{a}}=0
$$

where $w_{a}$ is the maximum displacement obtained from (120). Since (120) is a function of space and time, the conditions for maximum displacement are

$$
\frac{\partial w}{\partial x}=0, \frac{\partial w}{\partial y}=0,\left(1+\epsilon^{2} \mu+\cdots\right) \frac{\partial w}{\partial \hat{t}}=0
$$

From the first and second terms of (120), we obtain the values $x_{a}$ and $y_{a}$ of $x$ and $y$ respectively at maximum displacement as

$$
x_{a}=\frac{\pi}{2 m}, y_{a}=\frac{\pi}{2 n}
$$

On evaluating (120) at maximum values of $x$ and $y$, we get

$$
w=\epsilon w_{2}^{(1)}+\epsilon^{3} w_{2(n)}^{(3)}+\cdots
$$

Let $\hat{t}_{a}$ be the value of $\hat{t}$ at maximum displacement and let us expand it asymptotically as

$$
\hat{t}_{a}=\hat{t}_{0}+\epsilon^{2} \hat{t}_{2}+\epsilon^{3} \hat{t}_{3}+\cdots
$$

On expanding the last term of (122), we get

$$
\left(1+\epsilon^{2} \mu+\cdots\right)\left[\begin{array}{c}
\epsilon\left\{w_{2(n), \hat{t}}^{(1)}\left(\hat{t}_{0}\right)+\left(\epsilon^{2} \hat{t}_{2}+\epsilon^{3} \hat{t}_{3}+\cdots\right) w_{2(n), \hat{t} \hat{t}}^{(1)}\left(\hat{t}_{0}\right)+\left(\epsilon^{2} \hat{t}_{2}+\epsilon^{3} \hat{t}_{3}+\cdots\right)^{2} w_{2(n), \hat{t} \hat{t} \hat{t}}^{(1)}\left(\hat{t}_{0}\right)+\cdots\right\}+ \\
\epsilon^{3}\left\{w_{2(n), \hat{t}}^{(3)}\left(\hat{t}_{0}\right)+\left(\epsilon^{2} \hat{t}_{2}+\epsilon^{3} \hat{t}_{3}+\cdots\right) w_{2(n), \hat{t} \hat{t}}^{(3)}\left(\hat{t}_{0}\right)\right\}+\cdots
\end{array}\right]=0
$$


where (126) is evaluated at $\hat{t}=\hat{t}_{0}$. On equating coefficients of powers of $\epsilon$, we get

$$
\begin{gathered}
O(\epsilon): w_{2(n), \hat{t}}^{(1)}\left(\hat{t}_{0}\right)=0 \\
O\left(\epsilon^{3}\right): \hat{t}_{2} w_{2(n), \hat{t} \hat{t}}^{(1)}\left(\hat{t}_{0}\right)+\mu w_{2(n), \hat{t}}^{(1)}\left(\hat{t}_{0}\right)=0 \\
\text { etc. }
\end{gathered}
$$

From (127), we get, using (48)

$$
w_{a}=\epsilon w_{2}^{(1)}\left(\hat{t}_{0}\right)+\epsilon^{3} w_{2(n)}^{(3)}\left(\hat{t}_{0}\right)+\cdots=2 B \epsilon+\frac{4 B^{3} \epsilon^{3}}{3 \Psi^{2}}\left[1+\frac{4 \psi^{2}}{3}\left\{\frac{\varphi_{20}\left(1+\cos \Omega \hat{t}_{0}\right)}{\psi^{2}-\Omega^{2}}+\frac{\varphi_{21}\left(1-\cos (\psi+\Omega) \hat{t}_{0}\right)}{\Omega(2 \Psi+\Omega)}\right\}\right]
$$

We shall however write (130) simply as

$$
w_{a}=C_{1} \epsilon+C_{3} \epsilon^{3}+\cdots
$$

where,

$$
C_{1}=2 B, C_{3}=\frac{4 B^{3}}{3 \psi^{2}}\left[1+\frac{4 \psi^{2}}{3}\left\{\frac{\varphi_{20}\left(1+\cos \Omega \hat{t}_{0}\right)}{\psi^{2}-\Omega^{2}}+\frac{\varphi_{21}\left(1-\cos (\Psi+\Omega) \hat{t}_{0}\right)}{\Omega(2 \Psi+\Omega)}\right\}\right]
$$

The work in [16] had earlier noted that the invocation of (121) should be preceded by a reversal of the series (131) in the form

$$
\epsilon=d_{1} w_{a}+d_{3} w_{a}^{3}+\cdots
$$

By substituting in (132) for $w_{a}$ from (133) and equating the coefficients of powers of $\epsilon$, we get

$$
d_{1}=\frac{1}{C_{1}}, d_{3}=\frac{-C_{3}}{C_{1}^{4}}
$$

The maximization (121) is now initiated through (134) to get the value of $w_{a}$ at dynamic buckling, namely $w_{d}$, as

$$
\begin{gathered}
{\left[\left(m^{2}+n^{2} \xi\right)^{2}+\left\{\left(\frac{m A}{1+\xi}\right)^{2}+n^{2} \xi r\right\}(1+\xi)^{2}\left(\frac{n^{2} \xi r-m^{2}}{\left(m^{2}+n^{2} \xi\right)^{2}}\right)-\lambda_{D}\left\{\frac{\alpha m^{2}}{2}+n^{2} \xi\left(1-\frac{\alpha}{2}\right)\right\}\right]^{3 / 2}} \\
=\frac{3}{\sqrt{2}} \lambda_{D} \bar{a} \epsilon\left\{\frac{\alpha m^{2}}{2}+n^{2} \xi\left(1-\frac{\alpha}{2}\right)\right\} \sqrt{R}
\end{gathered}
$$

where,

$$
R=\left[1+\frac{4 \psi^{2}}{3}\left\{\frac{\varphi_{20}\left(1+\cos \Omega \hat{t}_{0}\right)}{\psi^{2}-\Omega^{2}}+\frac{\varphi_{21}\left(1-\cos (\psi+\Omega) \hat{t}_{0}\right)}{\Omega(2 \psi+\Omega)}\right\}\right]
$$

and $\mathrm{R}$ depends on $\lambda_{D}$ through $\Psi$ and $\Omega$. In an earlier paper [17], the static buckling load $\lambda_{S}$ and classical buckling load $\lambda_{C}$ of the same structure were respectively given by

$$
\begin{gathered}
{\left[\left(m^{2}+n^{2} \xi\right)^{2}+\left\{\left(\frac{m A}{1+\xi}\right)^{2}+n^{2} \xi r\right\}(1+\xi)^{2}\left(\frac{n^{2} \xi r-m^{2}}{\left(m^{2}+n^{2} \xi\right)^{2}}\right)-\lambda_{S}\left\{\frac{\alpha m^{2}}{2}+n^{2} \xi\left(1-\frac{\alpha}{2}\right)\right\}\right]^{3 / 2}} \\
=\frac{3 \sqrt{3}}{2} \lambda_{S}(\bar{a} \epsilon)\left\{\frac{\alpha m^{2}}{2}+n^{2} \xi\left(1-\frac{\alpha}{2}\right)\right\} \sqrt{Q_{1} Q_{7}}
\end{gathered}
$$

(for $Q_{1}, Q_{7}$ as there defined)

and

$$
\lambda_{C}=\frac{(1+\zeta)^{2}+\frac{A^{2}(1+\zeta r)}{(1+\zeta)^{2}+\zeta r(1+\zeta)^{2}}}{\frac{\alpha}{2}+\left(1-\frac{\alpha}{2}\right) \zeta}
$$


where,

$$
\zeta=\xi n^{2} \text { and for } m=1
$$

Using (136) and (138), we can relate the dynamic buckling load $\lambda_{D}$ and static buckling $\lambda_{S}$ to get

$$
\left[\frac{\left(m^{2}+n^{2} \xi\right)^{2}+\left\{\left(\frac{m A}{1+\xi}\right)^{2}+n^{2} \xi r\right\}(1+\xi)^{2}\left(\frac{n^{2} \xi r-m^{2}}{\left(m^{2}+n^{2} \xi\right)^{2}}\right)-\lambda_{D}\left\{\frac{\alpha m^{2}}{2}+n^{2} \xi\left(1-\frac{\alpha}{2}\right)\right\}}{\left(m^{2}+n^{2} \xi\right)^{2}+\left\{\left(\frac{m A}{1+\xi}\right)^{2}+n^{2} \xi r\right\}(1+\xi)^{2}\left(\frac{n^{2} \xi r-m^{2}}{\left(m^{2}+n^{2} \xi\right)^{2}}\right)-\lambda_{S}\left\{\frac{\alpha m^{2}}{2}+n^{2} \xi\left(1-\frac{\alpha}{2}\right)\right\}}\right]^{3 / 2}=\frac{\sqrt{6}}{3}\left(\frac{\lambda_{D}}{\lambda_{S}}\right) \sqrt{\frac{R}{Q_{1} Q_{7}}}
$$

\section{Analysis of Results}

The results obtained for any mode $\mathrm{m}$ (m odd), namely (136), (138) and (141) are implicit in nature and provide very useful and simple formulae for determining the dynamic buckling load of the structure that is pressurized by a step load. As equation (141) clearly shows, the results are such that we can relate the dynamic buckling load $\lambda_{D}$ to its static equivalent $\lambda_{S}$ and that relationship is independent of the imperfection parameter $\epsilon$. The mathematical implication is that a knowledge of one of these two buckling loads automatically leads to determining the other buckling load without the labour of repeating the arduous procedure all over for different imperfections. The results are however asymptotic and so, are guided by the relative smallness of the magnitude of the imperfection magnitude in relation to unity.

\section{Conclusion}

By using perturbation and asymptotic procedures in this paper, we have been able to analytically determine the dynamic buckling load of an imperfect finite toroidal shell segment that was pressurized by a step load. The inherent stress-free, time-independent but continuously differentiable imperfection was expanded in a Fourier series and, in the final analysis, an implicit formula for determining the dynamic buckling load was obtained. A significant contribution is that it is possible to relate the dynamic buckling load to its static equivalent and that relationship is independent of the imperfection. Thus, if any of these two buckling loads is known, then, we can automatically evaluate the other buckling load without the labour of repeating the arduous procedure all over for different imperfection parameters.

\section{References}

[1] Stein, M. and McElman, J. A. (1965), Buckling of segments of toroidal shells, AIAA Jnl., 3, 1704.

[2] Hutchinson, J. W. (1967), Initial post buckling behavior of toroidal shell segment, In. J. Solids Struct. 3, 97-115.

[3] Oyesanya, M. O. (2002), Asymptotic analysis of imperfection sensitivity of toroidal shell segment modal imperfection, J. Nigerian Ass. Maths. Physics, 6, 197-2006.
[4] Oyesanya, M. O. (2005), Influence of extra terms on asymptotic analysis of imperfection sensitivity of toroidal shell segment with random imperfection, Mechanics Research Communications, 32, 444-453.

[5] Lockhart, D. and Amazigo, J. C. (1975), Dynamic buckling of externally pressurized imperfect cylindrical shells, J. of App. Mech. 42 (2), 316-320.

[6] Ganaparthi, M., Gupta, S. S. and Patel, B. P. (2005), Nonlinear axisymmetric dynamic buckling of laminated angle-ply composite spherical caps, Composite Structures, 59, 89-97.

[7] Kolakowski, Z. (2007), Semi-analytical method for the analysis of the interactive buckling of thin-walled elastic Structures in the second order approximation, Int. J. Solids Struct., 2790-3779.

[8] Kolakowski, Z. and Mania, R. J. (2013), Semi-analytical method versus the FEM for analyzing the local post-buckling of thin-walled composite structures, Composite Structures, 97, 99-106.

[9] Kubiak, T. (2013), Static and dynamic buckling of thin-walled plates structures, Springer-Verlag London, UK.

[10] Magnucki, K., Paczos, P. and Kasprzak, J. (2010), Elastic buckling of cold-formed Thin-walled channel beams with drop flanges, J. of Structural Engrg. 136 (7), 886-896.

[11] Tetter, A. and Kalakowski, Z. (2013), Coupled dynamic buckling of Thin-walled composite columns with open crosssection, Composite Structures, 95, 28-34.

[12] Kriegesman, B., Mohle, M. and Rolfes, R. (2015), Sample size dependent probabilistic design of axially compressed cylindrical shells, Thin-Walled Structures, 96, 256-268.

[13] $\mathrm{Hu}, \mathrm{N}$. and Burgueno, R. (2014), Elastic post buckling response of axially loaded cylindrical shells with seeded geometric imperfection design, Thin-Walled Structures, 74, 222-231.

[14] Hutchinson, J. W. and Budiansky, B. (1966), Dynamic buckling estimates, AIAA J., 4, 525-530.

[15] Amazigo, J. C. and Ette, A. M. (1987), On a two-small parameter nonlinear differential equation with application to dynamic buckling, J. of Nigerian math. Soc. 6, 91-102.

[16] Amazigo, J. C. (1971), Buckling of stochastically imperfect columns on nonlinear elastic foundations, Quart. Appl. Math., 31 (1), 403-409.

Ette, A. M., Chukwuchekwa, J. U., Udo-Akpan, I. U. and Ozoigbo, G. E. (2019), On the normal response and buckling of a toroidal shell segment pressurized by a static compressive load, IJMTT, 65 (10), 15-26. 